\title{
Multiseptate Gallbladder. A Case Report.
}

\author{
Zamira Shabani ${ }^{1 *}$, Leart Berdica ${ }^{2}$, Arketa Pllumi ${ }^{1}$, Bledar Shega ${ }^{3}$
}

Received: 08 October 2021 / Accepted: 03 November 2021 / Published online: 20 January 2022

This article is published with open access at https://journal.astes.org.al

(C) The author(s) 2022. \& Copyright (C) 2022, the Albanian Society for Trauma and Emergency Surgery

(c) The Albanian Journal of Trauma and Emergency Surgery is an Open Access Journal. All articles are distributed under the terms of the Creative Commons Attribution Non-Commercial License: http://creativecommons.org/licenses/by-nc/4.0/) which permits unrestricted non-commercial use, distribution, and reproduction in any medium provided the original work is properly cited.

\begin{abstract}
Introduction. Multiseptate gallbladder is a rare congenital malformation of the gallbladder. Septate gallbladder is a rare congenital anomaly. It is anatomical malformations affecting its shape, size, number, and position.

Patients with usually admits to the emergency department with differential symptoms such as right upper abdominal pain, nause and vomiting, and abdominal complaints.

The aim of this case report is to present a case diagnosed with multiseptated gallbladder.

Case presentation. This case was diagnosed cause of the symptomatic clinic and with abdominal ultrasound such multiseptated gallbladder without stones. The case underwent surgical intervention for cholecystectomy. After the surgery intervention the material was sent for the histopathological diagnosis.
\end{abstract}

Conclusions. We recommend cholecystectomy in such cases due to possible complications in the future. In our case we evidence that the clinical story of patients is determinant for the treatment.

Keywords: Multiseptate gallbladder, abdominal ultrasound, cholecystitis, malformation, pain,

\section{Introduction}

Septate gallbladder is among the malformations that has not been well documented. Multiseptate gallbladder is a congenital malformation, the embryologic mechanism has still not been explained. [1]

Septate gallbladder is characterized by the presence of a septum or more septum that divides the gallbladder into two or more chambers. When the septum dividing the gallbladder lies longitudinally, it is called bilobed gallbladder, and when there is a transverse septum separating the fundus from the rest of the gallbladder, it is called an hour-glass gallbladder.

Original article, no submission or publication in advance or in parallel

\section{* Corresponding author:}

Asc. Prof. Dr. Zamira Shabani MD, Ph.D.,

$\bowtie$ zamira.shabani@unishk.edu.al

1 Department of Nursing, Faculty of Natural Sciences, "Luigj Gurakuqi" University, Shkodra, ALBANIA

2 Department of Pathology, University of Medicine of Tirana, ALBANIA

3 Department of Surgery, Regional Hospital of Shkodra, ALBANIA
Usually, it is asymptomatic or discovered accidentally during the evaluation of jaundice or at postmortem examination, but at times it may result in pitfalls at ultrasound imaging, causing a false-positive diagnosis of gallstones. Rarely, however, septate gallbladder causes recurrent attacks of abdominal pain or become complicated by cholelithiasis. [2]

Multiseptate gallbladder is a congenital abnormality categorized as a gallbladder shape variant with some 20 cases reported thus far in the literature. Clinical presentation may be highly variable, ranging from asymptomatic to chronic pain in the right upper quadrant, cholecystitis, and even pancreatitis. It may be associated with other bile duct abnormalities such as choledochal cyst, ectopic gallbladder or anomalous biliopancreatic junction. [3]

Robert Gross in 1936, collect from the literature the isolated reports and conduct a study where he illustrates 148 cases with different types of abnormalities of the gallbladder. A bilobed gallbladder has been occasionally described in man. This may have the form of a single organ, divided by an internal central septum, or more often has a $\mathrm{V}$ shape (with the two cavities joined only at their junction with the cystic duct). [4]

Patel at al. 2008 in their paper conclude the need for complete removal of gallbladder, as a remnant could be a 
good seat for development of stone in future and subsequent recurrence of symptoms.[5]

Lopez 2017 refer that ultrasound, particularly contrastenhanced sonography, should be considered as the "gold standard" technique for the diagnosis of gallbladder conditions. Given that its outcome is usually benign, multiseptate gallbladder should not be considered as an indication for cholecystectomy except when associated with cholecystopancreatitis, diagnostic doubt or disabling chronic pain. [6]

\section{Case Report}

This report describes one case with recurrent attacks of abdominal pain who was found to have a septate gallbladder on abdominal ultrasound and confirmed with surgery and biopsy. A 20-year-old female presented to the family doctor on April 2021, with recurrent attacks in the right upper quadrant, costal and abdominal intermittent pain, about a year's duration. The pain was colicky in nature and sometimes associated with vomiting and fever. The pain was more frequent recently due to fasting in the period of Ramadan. The pain started about an hour after eating and was hardly relieved with analgesics like anti-inflammatory non steroids. There was no history of sickle cell disease or any other blood disorder. On physical examination, there was no pallor/jaundice and the vitals were stable. On palpation, the abdomen was soft and non-tender. Examination revealed a healthy young girl with no other abnormalities. Her laboratory blood tests, liver function tests and electrolytes were normal. Abdominal ultrasound (Figure 1) revealed a multiseptated gallbladder with normal wall thickness.

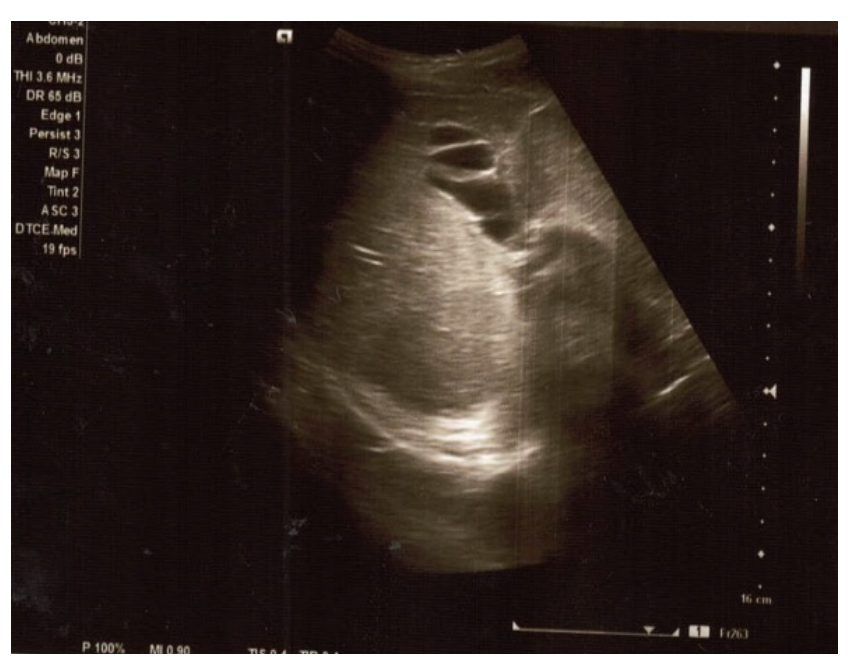

Figure 1 Ultrasound image of the multiseptal Gallbladder

The S-shaped bladder separated by 3 septa was identified. The longitudinal diameter of the gallbladder was $45 \mathrm{~mm}$ and horizontal diameter about $20 \mathrm{~mm}$. The patient underwent laparotomy surgery. In the operative field, intrahepatic cholecystitis was observed, with thin walls, irregular contours, without gallstones. With adhesions to the omentum and small intestine. Ligature is evident and cut between the ligament arteries and the cystic duct. During the intervention, a thin cystic duct was found, a gallbladder without calculates inside. She underwent laparotomy cholecystectomy. After the intervention, the removed gallbladder is dissected and 3 intraluminal septa are observed with small communication spaces between them. The removed gallbladder is sent for hystopathological examination. In the examination with the naked eye in the field of operation no other accompanying anomalies were noticed. Her condition improved after the operation and was discharged home on the third postoperative day. Histology of the gallbladder showed a multi septate gallbladder with three septa, confirmed as: chronic cholecystitis with cholesterolosis, with mucosal hyperplasia, mild dysplasia, focally with adenomyosis. (See figures: 2, 3).

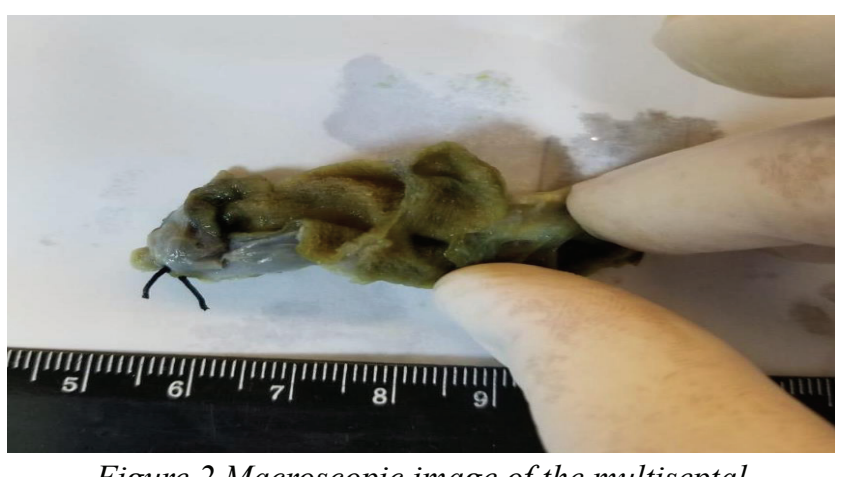

Figure 2 Macroscopic image of the multiseptal Gallbladder

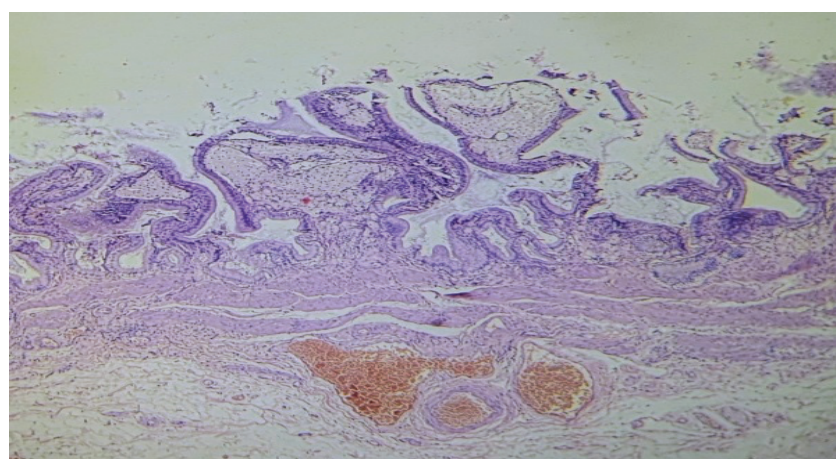

Figure 3 Histopathology examination - Chronic cholecystitis, polypoid cholesterolosis.

\section{Discussion}

Clinically, multiseptate gallbladder can be asymptomatic but most of patient present with biliary pain or colicky abdominal pain, usually localized in the epigastrium or right upper quadrant, sometimes irradiated in the back near the right scapula. Authors report symptomatic cases complicated by the presence of biliary sludge, stones and also cholecystitis. The absence of these complication lead to delay in diagnosis and surgical treatment. [7]

A similar case such ours was reported by Raina et al. [7] and Hendahewa et al. on 2021, they reported a 18-yearold female that was referred by a general practitioner to 
the surgical outpatients with a complaint of on and off right upper quadrant pain from the past 12 months. In this case cholecystectomy was the choice of treatment for the symptomatic patients. [8]

Also, another similar case was reported by Singh et al. [9] in 2020. They reported a 49-year-old woman with the complaint of intermittent right upper quadrant abdominal pain for the last few months. The abdominal pain was mild and no radiating with no associated nausea, vomiting, or skin discoloration, and without clear exacerbating or relieving factors.

This case was diagnosed with multiseptate gallbladder and was referred to a surgeon for cholecystectomy. The authors referred that the surgery has not been performed yet. [9]

In any clinical case we must listen carefully to the patient's history and pay attention to his complaints. Often, they are determinants in the pathology treatment protocol. Often, we are dealing with the same diagnosis but the clinic and treatment may be different. The result always depends on the cooperation between the patient and the family doctor, imaging, surgeon and morpho-pathologist.

\section{Conclusions:}

We recommend cholecystectomy in such cases due to possible complications in the future. In our case we evidence that the clinical story of patients is determinant for the treatment.

Author Contributions: Z.SH. was responsible for the diagnostic procedures and wrote the manuscript. A.P. made the ultrasound diagnosis. B.SH. was responsible for the surgery treatment. L.B. was responsible for the histopathological diagnosis. All authors have read and agreed to the published version of the manuscript.

\section{Declaration of Conflicting Interests and Ethics;}

The authors declare no conflict of interest. This research study complies with research publishing ethics. The scientific and legal responsibility for manuscripts published in Albanian Journal of Trauma and Emergency Surgery AJTES, belongs to the author(s).

Acknowledgements: None.

\section{References:}

1. Adear, H, and Y Barki. "Multiseptate gallbladder in a child: incidental diagnosis on sonography." Pediatric radiology vol. 20, 3 (1990): 192. doi:10.1007/BF02012972

2. Al-Salem, A. H., Issa, H., \& Naserullah, Z. Septate gallbladder: a report of two cases. Annals of Saudi medicine, 22 (5-6) (2002):351-353. https://doi.org/10.5144/02564947.2002.351

3. Wanaguru, Dylan et al. "Multiseptate gallbladder in an asymptomatic child." Case reports in gastrointestinal medicine vol. 2011 (2011): 470658. doi:10.1155/2011/470658

4. Gross Re. Congenital anomalies of the gallbladder: a review of one hundred and forty-eight cases, with report of a double gallbladder. Arch Surg. 1936, 32(1):131-162. https://doi. org/10.1001/archsurg.1936.01180190134008

5. Patel, Nitin R et al. "Septate gallbladder in the laparoscopic era." Journal of minimal access surgery vol. 4,1 (2008): 20-2. doi:10.4103/0972-9941.40994

6. Honrubia López, Raúl et al. "Multiseptate gallbladder: a rare ultrasonographic finding." Revista espanola de enfermedades digestivas : organo oficial de la Sociedad Espanola de Patologia Digestiva vol. 109,7 (2017): 527.

7. Rivera-Troche, Enid $\mathrm{Y}$ et al. "Multiseptate gallbladder." Journal of gastrointestinal surgery : official journal of the Society for Surgery of the Alimentary Tract vol. 13,9 (2009): 1741-3. doi:10.1007/s11605-009-0880-0.

8. Devisha Raina, and Rasika Hendahewa. "A Case Report on Multiseptate Gallbladder Clinical Manifestation and Treatment". Indian Journal of Case Reports, vol. 7, no. 1, Feb. 2021, pp. 40-41, doi:10.32677/IJCR.2021.v07.i01.013.

9. Singh AD, Simons-Linares CR, Chahal P. A dilated common bile duct with "atypical" gallbladder. Gastroenterology. 2020; 159: e6-7. 